\title{
LINEAR STABILITY ANALYSIS OF CONVECTIVE FLOW IN PIPE DUE TO RADIALLY DISTRIBUTED HEAT SOURCES
}

\author{
Valentina Koliskina, Andrei Kolyshkin \\ Riga Technical University, Latvia \\ v.koliskina@gmail.com, andrejs.koliskins@rtu.lv
}

\begin{abstract}
A steady convective flow of a viscous incompressible fluid is considered in a tall vertical cylindrical pipe due to internal heat sources of the form $Q=A+B r$, where $A$ and $B$ are constants and $r$ is the radial coordinate. Models of thermal convection with non-uniform internal heat generation are used in many applications. Examples include absorption of incidental radiation or zeroth-order chemical reactions. The pipe is closed so that the total fluid flux through the cross-section of the pipe is zero. The corresponding system of Navier-Stokes equations under the Boussinesq approximation is solved analytically. Linear stability of the steady convective flow is analyzed using the method of normal modes. The corresponding eigenvalue problem for the system of ordinary differential equations is solved numerically using the collocation method based on the Chebyshev polynomials. In the present paper we restrict ourselves only to asymmetric perturbations for the first azimuthal mode, since experiments for the case of heat sources of constant density have shown that it is the most unstable mode. It is found that even for Prandtl numbers smaller than 0.8 there exist two separate branches of the marginal stability curve. One of the branches is associated with the hydrodynamical mechanism due to the presence of an inflection point in the base flow velocity profile, while the second one is related to the instability in the form of thermal waves that propagate downstream with sufficiently large phase speed. It is found that the Prandtl number and both negative and positive B values have a destabilizing influence on the flow.
\end{abstract}

Keywords: linear stability, convective motion, heat sources.

\section{Introduction}

Convective flows in circular pipes often occur in applications. Linear stability of a steady convective motion caused by a vertical temperature gradient is investigated in [1-3]. It is shown that the most unstable mode is the azimuthal mode with the wave number $n=1$.Linear stability problem is solved in [4] for the case of heated bottom and cooled top and finite aspect ratio. Calculations show that marginal stability curves have a rather complicated shape (depending on the values of the parameters multiple modes can appear). A similar problem is analysed in [5], where the combined effect of differential heating and internal heat generation is investigated.

Linear stability analysis of a convective flow in a tall vertical pipe due to heat sources with constant density uniformly distributed within the fluid is considered in [6]. The role of the Prandtl number of the stability characteristics of the flow is analysed. Later a similar problem is solved for the case, where the heat sources are distributed within the fluid in accordance with the Arrhenius' law (see the solution for the circular pipe in [7] and for the annulus in [8]).

In the present paper we consider the linear stability problem of a steady convective motion in a tall vertical pipe. The steady flow is caused by heat sources with the density linearly dependent on the radial coordinate. Such a distribution can be used to model absorption of incidental radiation. Base flow solution is obtained analytically from the Navier-Stokes equations under the Boussinesq approximation. Both radially increasing and decreasing heat sources are analysed. Numerical calculations are performed for different values of the parameters of the problem.

\section{Mathematical formulation of the problem}

Convective flow in a vertical pipe is described by the system of the Navier-Stokes equations in the Boussinesq approximation:

$$
\begin{aligned}
\frac{\partial \vec{v}}{\partial t}+G r(\vec{v} \nabla) \vec{v} & =-\nabla p+\Delta \vec{v}+T \vec{k}, \\
\frac{\partial T}{\partial t}+G r(\vec{v} \nabla T) & =\frac{1}{P r} \Delta T+\frac{Q(r)}{P r} \\
\Delta \vec{v} & =0,
\end{aligned}
$$


where $\vec{v}-$ the velocity vector;

$T$ - the temperature;

$p$ - pressure;

$Q(r)$ is the density of the internal heat sources;

$\vec{k}=\{0,0,1\}, G r=g \beta Q_{0} R^{4} /\left(\mu^{2} k \rho c_{p}\right)$ is the Grashof number

$\operatorname{Pr}=\mu / k$ is the Prandtl number.

All the variables are made dimensionless by choosing the following scales of length $(R)$, time $\left(R^{2} / \mu\right)$, velocity $\left(g \beta Q_{0} R^{4} /\left(k \mu \rho c_{p}\right)\right)$, temperature $\left(Q_{0} R^{2} /\left(k \rho c_{p}\right)\right)$ and pressure $\left(g \beta Q_{0} R^{3} /\left(k c_{p}\right)\right)$, respectively. We have used the following notations: $R$ - radius of the pipe, $\mu$ - kinematic viscosity of the fluid, $\beta$ - coefficient of the thermal expansion, $\rho$ - density of the fluid, $k$ - coefficient of the thermal conductivity, $c_{p}$ - heat capacity and $g$ - acceleration due to gravity. The density of the internal heat sources is assumed to be of the form $Q(r)=Q_{0}(1+\alpha r)$.

The system (1)-(3) admits the following steady solution:

$$
\vec{v}_{0}(r)=\left(0,0 W_{0}(r)\right), T=T_{0}(r), p_{0}=A z+B,
$$

where $A$ and $B$ - constants.

Substituting (4) into (1)-(3) we obtain the following system of ordinary differential equations for the functions $W_{0}(r)$ and $T_{0}(r)$ :

$$
\begin{gathered}
\frac{d^{2} T_{0}}{d r^{2}}+\frac{1}{r} \frac{d T_{0}}{d r}+1+\alpha r=0, \\
\frac{d^{2} W_{0}}{d r^{2}}+\frac{1}{r} \frac{d W_{0}}{d r}+T_{0}+C=0,
\end{gathered}
$$

where $C$ - the separation constant.

The boundary conditions have the form

$$
T_{0}^{\prime}(0)=0, T_{0}(1)=0, W_{0}^{\prime}(0)=0, W_{0}(1)=0 .
$$

We assume that the pipe is infinitely long, but closed. Thus, the total fluid flux through the crosssection of the pipe is equal to zero:

$$
\int_{0}^{1} r W_{0}(r) d r=0
$$

The solution to (5)-(8) is

$$
\begin{gathered}
W_{0}(r)=\frac{1}{192}+\frac{r^{4}}{64}-\frac{r^{2}}{48}+\alpha\left(\frac{r^{5}}{255}-\frac{2 r^{2}}{315}\right)+\frac{\alpha}{525}, \\
T_{0}(r)=\frac{1-r^{2}}{4}+\frac{\alpha}{9}\left(1-r^{3}\right) .
\end{gathered}
$$

The case $\alpha=0$ corresponds to constant density of internal heat sources. It is seen from (9) and (10) how the non-uniformity $(\alpha \neq 0)$ of the density of the heat sources affects both steady temperature and velocity distributions. The graphs of the steady velocity distribution (9) and the temperature distribution (10) versus $r$ for different values of $\alpha$ are shown in Figs. 1 and 2.

Consider a perturbed flow of the form

$$
\vec{v}=W_{0}(r) \vec{k}+\vec{v}^{\prime}(r, \theta, t), T=T_{0}(r)+T^{\prime}, p=p_{0}+p^{\prime},
$$

where $\quad \vec{v}^{\prime}, T^{\prime}, p^{\prime}-$ small perturbations. 


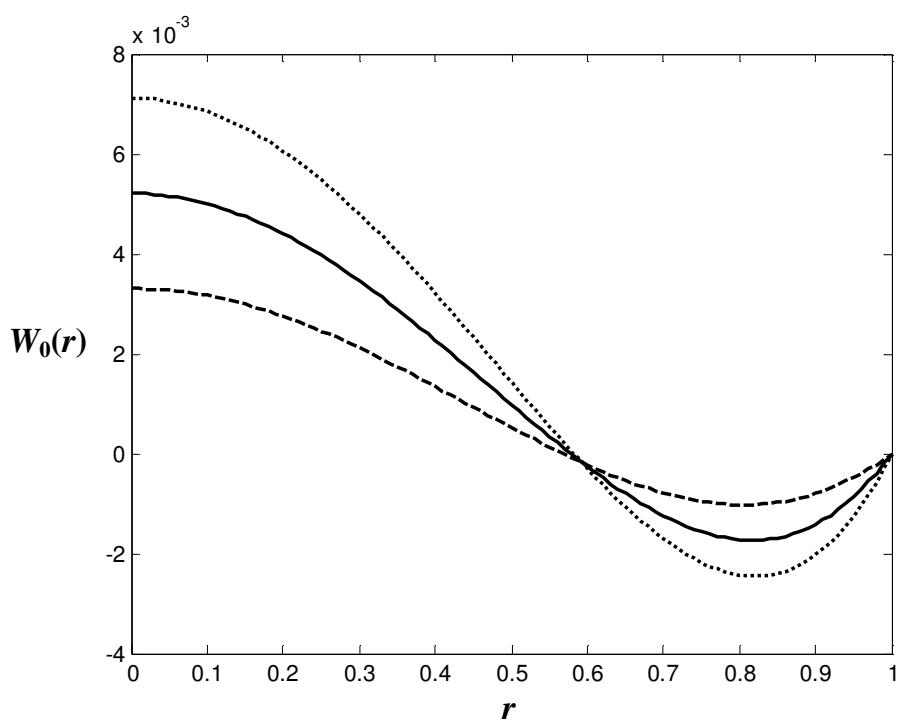

Fig. 1. Base flow velocity distribution for three values of $\alpha=1,0$ and -1 (dotted line, solid line and dashed line, respectively)

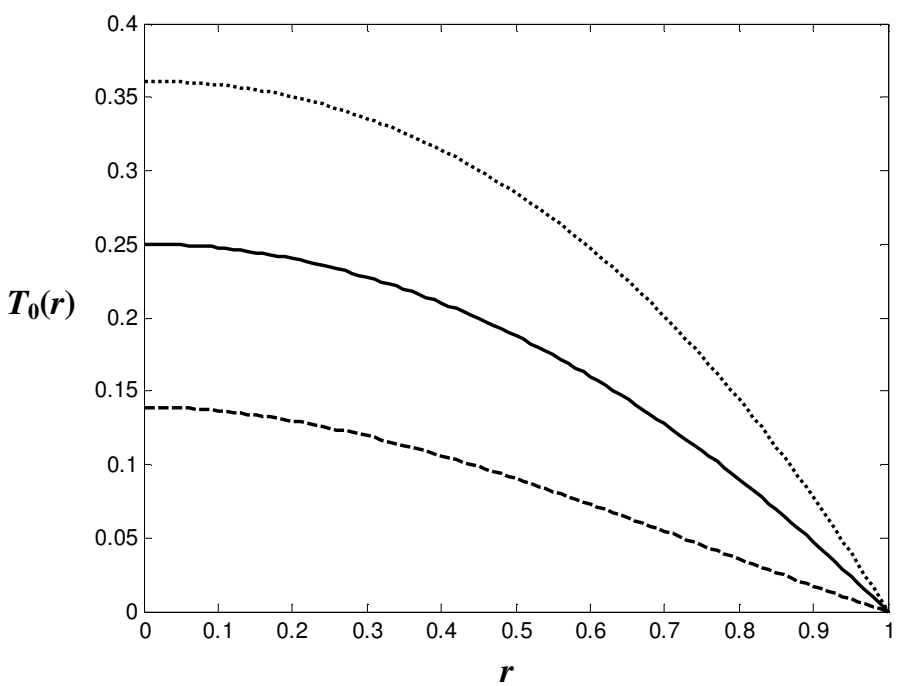

Fig. 2. Base flow temperature distribution for three values of $\alpha=1,0$ and -1 (dotted line, solid line and dashed line, respectively)

Substituting (11) into (1)-(3) and linearizing the resulting system of equations in the neighbourhood of the base flow (9), (10), we obtain the linearized system of partial differential equations. Using the method of normal modes, we represent the perturbed quantities in the form

$$
\begin{aligned}
& v_{r}^{\prime}(r, \theta, t)=u(r) e^{-\gamma+i s x+i n \theta}, \\
& v_{\theta}^{\prime}(r, \theta, t)=v(r) e^{-\gamma+i s x+i n \theta}, \\
& v_{z}^{\prime}(r, \theta, t)=w(r) e^{-\gamma+i s x+i n \theta}, \\
& T^{\prime}(r, \theta, t)=\vartheta(r) e^{-\gamma+i s x+i n \theta}, \\
& p^{\prime}(r, \theta, t)=q(r) e^{-\gamma+i s x+i n \theta},
\end{aligned}
$$

where $u(r), v(r), w(r), \vartheta(r), q(r)$ - the amplitudes of the normal perturbations; $\gamma$-complex eigenvalue;

$s$ and $n-$ the axial and azimuthal wave numbers, respectively. 
Substituting (11)-(16) into (1)-(3) and performing linearization procedure we obtain the system of ordinary differential equations for the amplitudes of the normal perturbations (the primes in the equations below represent the derivatives with respect to $r$ :

$$
\begin{gathered}
-\gamma u+i s G r W_{0} u=-q^{\prime}+u^{\prime \prime}+\frac{u^{\prime}}{r}-\frac{\left(1+n^{2}\right) u}{r^{2}}-s^{2} u-\frac{2 i n v}{r^{2}}, \\
-\gamma+i s G r W_{0} v=-\frac{i n q}{r}+v^{\prime \prime}+\frac{v^{\prime}}{r}-\frac{\left(1+n^{2}\right) v}{r^{2}}-s^{2} v-\frac{2 i n u}{r^{2}}, \\
-\gamma w+G r\left(u W_{0}^{\prime}+i s W_{0} w\right)=-i s q+w^{\prime \prime}+\frac{w^{\prime}}{r}-\frac{n^{2}}{r^{2}} w-s^{2} w-\vartheta, \\
-\gamma \vartheta+G r\left(u T_{0}^{\prime}+i s W_{0} \vartheta\right)=\frac{1}{\operatorname{Pr}}\left(\vartheta^{\prime \prime}+\frac{\vartheta^{\prime}}{r}-\frac{n^{2}}{r^{2}} \vartheta-s^{2} \vartheta\right), \\
u^{\prime}+\frac{u}{r}+\frac{i n v}{r}+i s w=0 .
\end{gathered}
$$

The boundary conditions at $r=1$ (at the solid boundary) are the same for all $n$ and have the form

$$
u(1)=0, v(1)=0, w(1)=0, \vartheta(1)=0, q^{\prime}(1)=0 .
$$

The boundary conditions at $r=0$ depend on the azimuthal wave number $n$. We consider only asymmetric perturbations with $n=1$ since this mode is the most unstable for the case of constant density of heat sources. The boundary conditions for $n=1$ are

$$
u(0)+i v(0)=0,2 u^{\prime}(0)+i v^{\prime}(0)=0, w(0)=0, \vartheta(0)=0, q(0)=0 .
$$

\section{Numerical results and discussion}

Collocation method is used in the paper for numerical solution of (17)-(23). The solution is sought in the form

$$
\begin{gathered}
u(x)=\sum_{m=0}^{N-1} a_{m} T_{m}(x), v(x)=\sum_{m=0}^{N-1} b_{m} T_{m}(x), w(x)=\sum_{m=0}^{N-1} c_{m} T_{m}(x), \\
\vartheta(x)=\sum_{m=0}^{N-1} d_{m} T_{m}(x), q(x)=\sum_{m=0}^{N-1} e_{m} T_{m}(x),
\end{gathered}
$$

where $x=2 r-1, T_{m}(x)=\cos (n \operatorname{arcos} x)$ is the Chebyshev polynomial of the first kind of order $m$; $N$ - the number of collocation points;

$a_{m}, b_{m}, c_{m}, d_{m}, e_{m}-$ unknown coefficients.

The collocation points are

$$
x_{j}=\cos \left(\frac{\pi j}{N-1}\right), j=0,1, \ldots, N-1 .
$$

Substituting (24)-(26) into (17)-(23) we obtain the generalized eigenvalue problem of the form

$$
(C-\gamma D) \vec{f}=0
$$

where $C$ and $D$-complex-valued matrices (the matrix $D$ is singular);

$$
\vec{f}=\left(a_{0} a_{1} \ldots a_{N-1} b_{0} b_{1} \ldots b_{N-1} \ldots e_{0} e_{1} \ldots e_{N-1}\right)^{T} .
$$

The method suggested in [9] is used to transform (27) to the non-singular generalized eigenvalue problem, which is solved numerically using Matlab.

Calculations are performed for the case $\operatorname{Pr}=0.79$ for three values of $\alpha$, namely, $\alpha=0,1,2$. The results are shown in Fig. 3. 


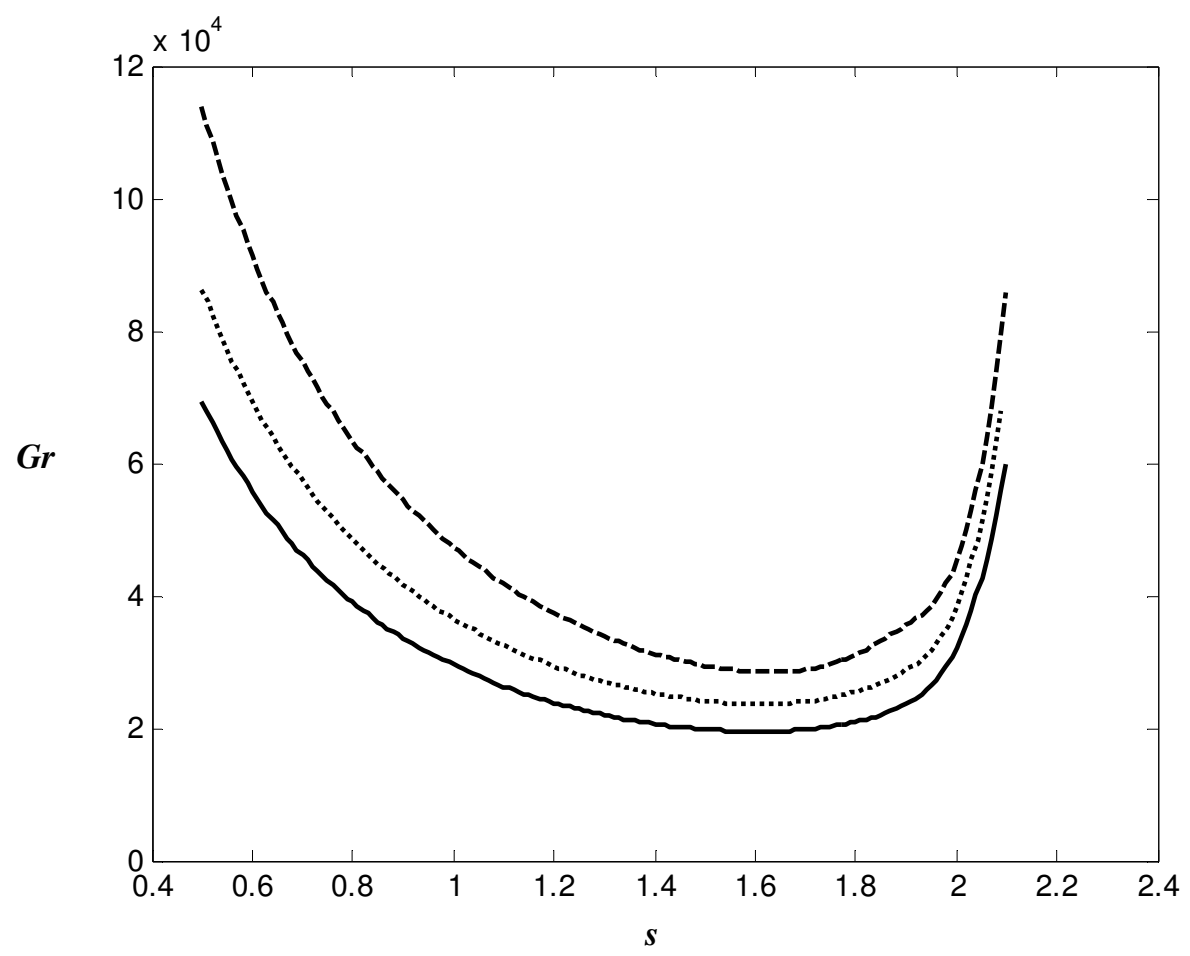

Fig. 3. Marginal stability curves for three values of $\alpha=0,1,2$ (dashed line, dotted line and solid line, respectively)

It is seen from the graph that the increase of the heat sources density in the radial direction (the increase in $\alpha$ ) has a destabilizing influence on the flow. The case of negative $\alpha$ is shown in Fig. 4, where the marginal stability curve for constant heat sources density is also plotted.

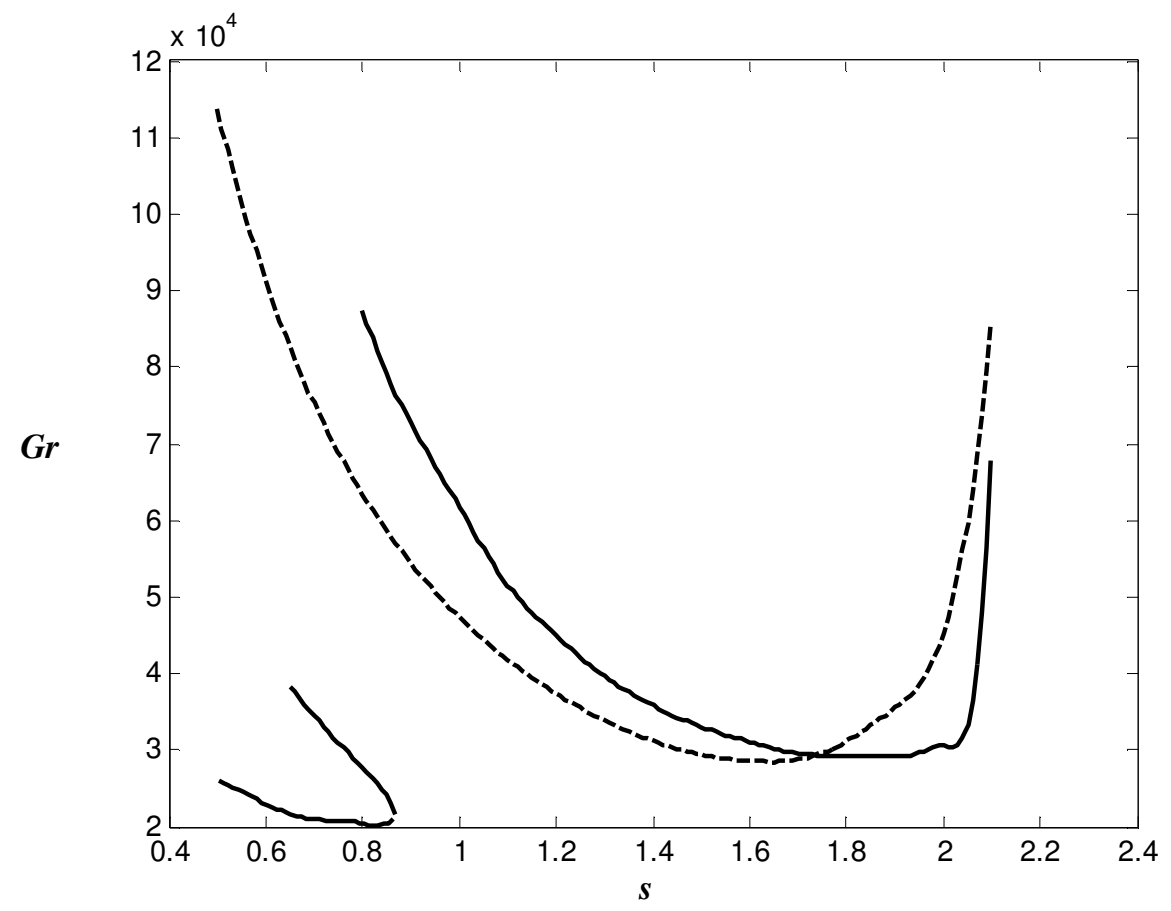

Fig. 4. Marginal stability curves for $\alpha=0$ (dashed line) and $\alpha=-1$ (solid lines)

It is interesting to note that the marginal stability curve for the case $\alpha=-1$ consists of two separate branches. In addition, the instability is associated with small wave number range. Marginal stability curve for the case $\alpha=-1, \operatorname{Pr}=3$ is shown in Fig. 5. 


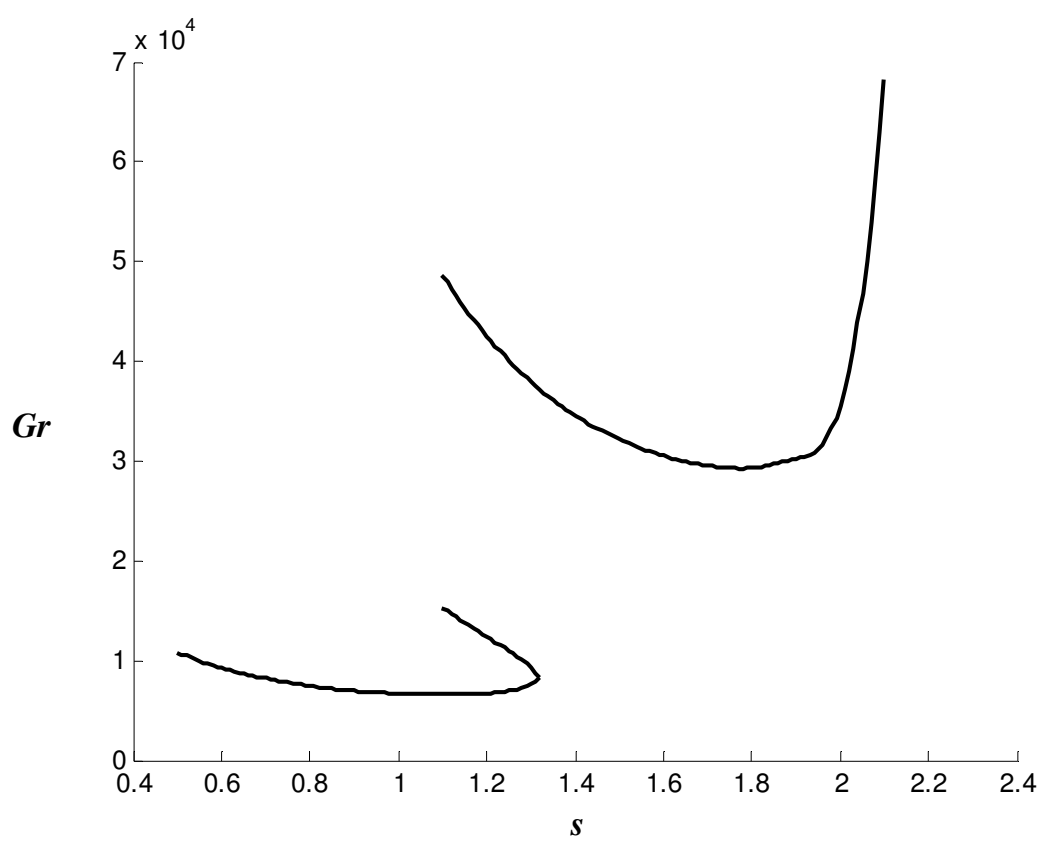

Fig. 5. Marginal stability curve for $\alpha=-1$ and $P r=3$

Comparing Figs. 4 and 5, we see that the instable branch of the marginal stability curve (corresponding to smaller wave numbers) lowers as the Prandtl number increases.

\section{Conclusions}

1. Non-uniformity of the heat source density has a destabilizing influence on the base flow.

2. Marginal stability curve for the case $\alpha=-1$ consists of two separate branches.

3. The increase of the Prandtl number destabilizes the flow.

\section{References}

[1] Yao L.S. Is a fully developed and non-isothermal flow possible in a vertical pipe? International Journal of Heat and Mass Transfer, vol. 30, 1987, pp. 707-716.

[2] Rogers B.B. and Yao L.S. Finite-amplitude instability of mixed-convection in a heated vertical pipe. International Journal of Heat and Mass Transfer, vol. 36, 1993, pp. 2305-2315.

[3] Su Y.C. and Chung J.N. Linear stability analysis of mixed-convection flow in a vertical pipe. Journal of Fluid Mechanics, vol. 422, 2000, pp. 141-166.

[4] Wang B.F., Ma D.J., Chen C., and Sun D.J. Linear stability analysis of cylindrical RayleighBenard convection. Journal of Fluid Mechanics, vol. 711, 2012, pp. 27-39.

[5] Wang B.F., Zhou L.,Wan Z.H., Ma D.J., and Sun D.J. Stability analysis of Rayleigh-Benard convection in a clynider with internal heat generation. Physical Review E, Jul 94(1-1):013108. doi: 10.1103/PhysRevE.94.013108. 2016.

[6] Kolyshkin A.A. and Vaillancourt R. Linear stability of a convective flow in a vertical pipe. In: Recent research in applied mathematics, 2010, pp. 44-48.

[7] Koliskina V., Kolyshkin A., Volodko I., and Kalis H. On the stability of a convective motion generated by a chemically reacting fluid in a pipe. AIP Conference Proceedings, 1738, 480028, 2016.

[8] Iltins I., Iltina M., Kolyshkin A. Convective stability of a chemically reacting fluid in annulus. Proceedings of the 7th International conference on computational methods for coupled problems in science and engineering, 2017, pp. 427-433.

[9] Gary J. and Helgason R. A matrix method for ordinary differential eigenvalue problems. Journal of Computational Physics, vol. 5, 1970, pp. 169-187. 